hep-th/0409050

UCLA/04/TEP-36

KIAS-P04036

\title{
Winding Strings and Decay of D-Branes with Flux
}

\author{
Michael Gutperle日 \\ Department of Physics and Astronomy, UCLA, Los Angeles, CA 90095-1547, USA \\ and \\ Piljin Yi目 \\ Institute for Advanced Study, Princeton, NJ 08540, USA \\ and \\ School of Physics, Korea Institute for Advanced Study \\ 207-43, Cheongryangri-Dong, Dongdaemun-Gu, Seoul 130-722, Korea
}

\begin{abstract}
We study the boundary state associated with the decay of an unstable D-brane with uniform electric field, $1>e>0$ in the string units. Compactifying the D-brane along the direction of the electric field, we find that the decay process is dominated by production of closed strings with some winding numbers; closed strings produced are such that the winding mode carries precisely the fraction $e$ of the individual string energy. This supports the conjecture that the final state at tree level is composed of winding strings with heavy oscillations turned on. As a corollary, we argue that the closed strings disperse into spacetime at a much slower rate than the case without electric field.
\end{abstract}

\footnotetext{
1 gutperle@physics.ucla.edu

2 piljin@kias.re.kr
} 


\section{Closed Strings and Unstable D-Branes with Electric Field}

Decay of unstable D-branes [1] has been studied extensively in the classical limit of open string theory yet our understanding remains limited. Most notably, whether and precisely how closed string degrees of freedom emerge from the decay remains an issue. For some of effort in searching for fundamental strings in the low energy pictures can be found in [2] [3] [4] [5]. Only in the limited setting of $1+1$ dimensions, a successful recovery of closed strings has been seen, thanks to reinterpretation of old $c=1$ matrix model as theory of many unstable D-branes [6] [7] [8] [9].

For more generic string theories, an important breakthrough came about two years ago, when A. Sen wrote down time-dependent boundary states describing decay of unstable D-branes [10][11]. This set a stage for more precise questions about evolution of unstable D-branes in fully stringy setting [12] 13] 14] 15] 16] 18] 19]. So far, however, many successful efforts in this regard have been aimed at understanding the homogeneous final state. For questions related to dynamical objects, such as lower dimensional D-branes, the low energy approach still proves much more powerful and also somewhat unexpectedly accurate. 3

One unusual property of this tachyonic system is that all fundamental degrees of freedom one starts with, namely those associated with open strings, will have to disappear eventually. This seems to raise a paradox since the dynamics involves no dissipation: all conserved quantities such as energy, momentum, and gauge charges must survive somehow. In particular, it is important to note that this has to be true even in the classical limit, $g_{s} \rightarrow 0$. We are accustomed to the idea that open string theory is self-contained at classical level and does not require presence of closed strings, however, and we are thus faced with quandary of where to attribute these conserved quantities after disappearance of all dynamical degrees of freedom available at tree level.

Through study of low energy approximation [4][21] [22], and also study of boundary states [10] [11], both at tree level, some effective degrees of freedom have been found to carry these conserved quantities. They are called tachyon matter [11] and string fluid [4]. Both carry energy and momentum, while the latter carry in addition the fundamental string charge induced by the electric field on the worldvolume. They can both be viewed

3 Perhaps one of the more beautiful examples of this can be found in the construction of low energy dynamics of solitonic D-branes, starting with the severely truncated low energy approximation of unstable D-branes [20]. 
as fluid in that their state is specified completely by the distribution and the flow of energy, momentum, and electric flux. Their equation of states are such that no pressure is present other than the tension along the flux lines [4] 22] 23].

For instance, the Hamiltonian for the tachyon matter (in the absence of string fluid) collapses to 21]

$$
\mathcal{H}=\sqrt{\left(\pi_{T}^{2}\right)\left(1+(\vec{\partial} T)^{2}\right)},
$$

where $\pi_{T}$ is the conjugate momentum to $T$. The resulting dynamics is that of a perfect fluid with density distribution $\pi_{T}$ and the rotationless velocity field $\partial_{i} T, i=1, \ldots, p$, on the unstable $\mathrm{D} p$-brane worldvolume. While tachyon matter is intriguing on its own, life becomes more interesting when electric field is turned on. The final state of the decay encodes fundamental string charge in the conjugate momentum $\pi_{i}$ of the gauge field, which is nothing but the conserved electric flux obeying the Gauss law, $\partial_{i} \pi_{i}=0$. The Hamiltonian with electric field is also pretty simple [四,

$$
\mathcal{H}=\sqrt{(\vec{\pi})^{2}+\pi_{T}^{2}+(\vec{P})^{2}+\left(\pi_{i} \partial_{i} T\right)^{2}}
$$

with the Poynting vector $P_{i}=F_{i k} \pi_{k}+\partial_{i} T \pi_{T}$. Again the equation of motion from this is fluidic, where the tachyon matter density, $\pi_{T}$, interacts with the string fluid density, $\pi_{i}$ [23].

As the name suggests, the string fluid behaves remarkably like a continuum of noninteracting Nambu-Goto strings [4] [5]. This prompted the speculation that the string fluid represents seed for formation of closed string as a coherent state of unstable open strings [4] 24]. Irrespective of such effort, on the other hand, a more conservative interpretation is possible in the classical limit. Note that the total energy of an unstable D-brane scales as $\sim 1 / g_{s}$, so with finite electric field, the amount of fundamental string charge per unit volume is enormous. This fact allows a macroscopic interpretation of the classical string fluid as a collection of large number of long winding strings [4]. At the end of decay process, nothing but closed strings would be left in full string theory, so this is interpretation is both natural and quite satisfying.

Once this macroscopic interpretation of string fluid is accepted, it still remains to attribute the energy and momentum of tachyon matter. Again in real string theory, the only degrees of freedom at the end are closed strings, so it is tempting to view tachyon matter again as large number of highly massive closed string modes. From inspection of low energy dynamics, furthermore, it is suggested that these high frequency modes of 
strings are not independent of winding strings but rather must be on top of the latter. Here, one must imagine the closed strings carrying both the winding mode and additional high frequency oscillation modes with the latter moving up and down along the length of strings [25].

Note that the conserved electric flux $\pi_{i}$ is different from the electric field strength $F_{0 i}$ in general. Assuming a static configuration, $P_{i}=0$, and also $\pi_{i} \partial_{i} T=0$ for the sake of simplicity, we have the relationship [22] 23],

$$
\pi_{i}=\mathcal{H} F_{0 i}
$$

and

$$
\pi_{T}=\mathcal{H} \sqrt{1-\sum\left(F_{0 i}\right)^{2}},
$$

which also tells us that $\sum\left(F_{0 i}\right)^{2}$ lies between 0 and 1 . Interpreting the conserved charge $\pi_{i}$ as the string charge, one finds that this fluid description would be consistent with the closed string picture if we have the following ratio,

$$
\frac{\text { string charge }}{\text { string energy }}=e \text {, }
$$

for individual closed strings [25], where a new notation is introduced for the size of electric field strength

$$
e \equiv \sqrt{\sum\left(F_{0 i}\right)^{2}}
$$

This picture has been tested by comparing the fluid motion (of low energy open string theory) to motion of many oscillatory Nambu-Goto strings [26]. Both static and dynamics properties have been seen to match precisely.

We arrive at the picture of final state of unstable D-brane decay as a large collection of aligned and stretched fundamental strings with some high frequency modes turned on. Motion of string fluid, encoded in $\pi_{i}$, represents low frequency undulation of this collection, while the tachyon matter captures the energy-momentum of high frequency modes moving up and down the same strings. In other words, the fluid picture in the low energy open string theory is just a coarse-grained view of some specific type of states involving many closed strings. This remarkable emergence of closed string picture may provide some hint on relationship between open strings and closed strings, and has been referred to as an open/closed duality [25]. The purpose of this note is to elevate this open/closed dual picture of the final state of D-brane decay to a fully stringy level. 
For this, we will ask how does the boundary state look like in the late time. One way to answer this is to analyze the decay product of the process, since we should be able to infer some properties of the boundary state by looking at irradiation of closed strings. In particular, contributions to the decay arises dominantly from the disk one point function, one can directly read off the composition of the late time boundary state from the distribution of emitted closed string modes. It is argued that in general one must consider multi-point disk amplitude to see complete decay of the brane, except the case of unstable D0. However, as we will see below, if one considers unstable D1-brane wrapped on a circle, it turns out that one point amplitude suffices in a manner quite similar to the case of unstable D0 case in noncompact spacetime [19].

\section{Boundary state}

Our conventions are as follows. We set $\alpha^{\prime}=1$ and define the mode expansion as

$$
\begin{aligned}
& X_{L}^{i}(z)=x_{L}^{i}-\frac{i}{2} p_{L}^{i} \ln (z)+\frac{i}{\sqrt{2}} \sum_{n \neq 0} \frac{1}{n} a_{n}^{i} z^{-n} \\
& X_{R}^{i}(\bar{z})=x_{R}^{i}-\frac{i}{2} p_{R}^{i} \ln (\bar{z})+\frac{i}{\sqrt{2}} \sum_{n \neq 0} \frac{1}{n} \bar{a}_{n}^{i} \bar{z}^{-n} .
\end{aligned}
$$

Where the worldsheet is a cylinder parameterized by $z=e^{\tau-i \sigma}$. The commutation relations for spacelike bosons are given by

$$
\begin{array}{ll}
{\left[x_{L}^{i}, p_{L}^{j}\right]=i \delta^{i j},} & {\left[a_{n}^{i}, a_{m}^{j}\right]=n \delta^{i j} \delta_{m+n},} \\
{\left[x_{R}^{i}, p_{R}^{j}\right]=i \delta^{i j},} & {\left[\bar{a}_{n}^{i}, \bar{a}_{m}^{j}\right]=n \delta^{i j} \delta_{m+n} .}
\end{array}
$$

If a bosonic coordinate $X^{i}$ is compactified on a circle of radius $R_{i}$, the left and right momenta take the form

$$
k_{L}^{i}=\frac{n^{i}}{R_{i}}+w_{i} R_{i}, \quad k_{R}^{i}=\frac{n^{i}}{R_{i}}-w_{i} R_{i},
$$

where $n^{i} \in Z$ is the KK momentum and $w_{i} \in Z$ is the winding number in the $i$-th direction.

A boundary state imposes the boundary conditions for open strings (ending on a Dbrane) on the closed string modes. The boundary state is useful since the sourcing of the closed string fields by the D-brane can be read off easily. The boundary conditions along 
the worldvolume directions of a D-brane in the presence of a magnetic field $b_{i j}$ are given by (the boundary of the worldsheet is located at $\tau=0$ on the cylinder).

$$
\left(g_{i j} \partial_{n} X^{j}+i b_{i j} \partial_{t} X^{j}\right)|B\rangle=0
$$

where $\partial_{n}=\partial_{\tau}, \partial_{t}=\partial_{\sigma}$. Inserting the mode expansion (2.1) then gives the following condition on the oscillators and zero modes

$$
\begin{aligned}
\left((g+b)_{i j} a_{n}^{j}+(g-b)_{i j} \bar{a}_{-n}^{j}\right)|B\rangle & =0, \quad n \in Z \\
\left(g_{i j}\left(p_{L}^{j}+p_{R}^{j}\right)+b_{i j}\left(p_{l}^{j}-p_{R}^{j}\right)\right)|B\rangle & =0
\end{aligned} .
$$

In the following we will focus on two (Euclidean) directions $i=1,2$ compactified on circles of radius $R_{1}, R_{2}$ respectively. The metric metric and magnetic field given by

$$
g_{i j}=\left(\begin{array}{ll}
1 & 0 \\
0 & 1
\end{array}\right), \quad b_{i j}=\left(\begin{array}{cc}
0 & -e \\
e & 0
\end{array}\right) .
$$

In this case the boundary state takes the form

$$
|B\rangle=C \sqrt{1+e^{2}} \exp \left(-\sum_{n>0} \frac{1}{n} a_{-n}^{i} M_{i j} \bar{a}_{-n}^{j}\right)|B\rangle_{0} .
$$

Where $C$ is a $e$ independent normalization factor and

$$
M_{i j}=\frac{(g-b)}{(g+b)}=\frac{1}{1+e^{2}}\left(\begin{array}{cc}
1-e^{2} & 2 e \\
-2 e & 1-e^{2}
\end{array}\right)
$$

is an orthogonal matrix.

The zero mode part $|B\rangle_{0}$ of the boundary state which solves (2.5) is given by

$$
\begin{array}{r}
|B\rangle_{0}=\sum_{w_{1}, w_{2}} \mid k_{L}^{1}=e R_{2} w_{2}+R_{1} w_{1}, k_{R}^{1}=e R_{2} w_{2}-R_{1} w_{1}, \\
\left.k_{L}^{2}=-e R_{1} w_{1}+R_{2} w_{2}, k_{R}^{2}=-e R_{1} w_{1}-R_{2} w_{2}\right\rangle .
\end{array}
$$

In the limit $R_{1} \rightarrow \infty$ only the zero mode sector $w_{1}=0$ survives. Note however that the winding $w_{2}$ along the $X^{2}$ modifies the momentum in the $X^{1}$ direction. For notational simplicity we will drop the index on the winding number $w_{2}$ and the circle radius $R_{2}$ and replace them by $w$ and $R$ respectively.

A open string field $X(\sigma)$ is located at $\tau=0$. The presence of the magnetic field modifies the OPE

$$
X^{i}\left(\sigma_{1}\right) X^{j}\left(\sigma_{2}\right)=-\frac{2}{1+e^{2}} \delta^{i j} \ln \left|e^{i \sigma_{1}}-e^{i \sigma_{2}}\right|+2 \frac{e}{1+e^{2}} \epsilon^{i j} \epsilon\left(\sigma_{1}, \sigma_{2}\right) .
$$


In particular a boundary vertex operator of the form $e^{i \sqrt{1+e^{2}} X^{1}}$ has conformal dimension one. Indeed this operator can be used to define an exactly marginal deformation of the boundary state $(2.7)$.

$$
|B(\lambda)\rangle=\exp \left(-2 \pi \lambda \oint \frac{d \sigma}{2 \pi} e^{i \sqrt{1+e^{2}} X^{1}(\sigma)}\right)|B\rangle
$$

The part of the boundary state which does not involve any oscillators in the $X^{1}$ direction can be evaluated by simply expanding the boundary deformation in $\lambda$ and using (2.10) to evaluate the correlators,

$$
|B(\lambda)\rangle_{0}=\sum_{n} \frac{(-2 \pi \lambda)^{n}}{n !} \prod_{i=1}^{n} \oint \frac{d \sigma_{i}}{2 \pi} \prod_{i<j}\left|e^{i \sigma_{i}}-e^{i \sigma_{j}}\right|^{2} e^{i n \sqrt{1+e^{2}} x_{1}}|B\rangle .
$$

Using the fact

$$
\prod_{i=1}^{n} \oint \frac{d \sigma_{i}}{2 \pi} \prod_{i<j}\left|e^{i \sigma_{i}}-e^{i \sigma_{j}}\right|^{2}=n !
$$

One can express (2.12) as

$$
|B(\lambda)\rangle_{0}=\frac{1}{1+\lambda e^{i \sqrt{1+e^{2}} x_{1}}}|B\rangle
$$

An analytic continuation $X^{1} \rightarrow i X^{0}$ turns the spacelike coordinate $X^{1}$ into a timelike coordinate $X^{0}$. The boundary state stays real if one also continues $e \rightarrow-i e$, this analytic continuation corresponds to turning on an electric field in the 0,2 directions instead of a magnetic field in the 1,2 direction. After analytic continuation to the timelike case (2.14) becomes

$$
|B(\lambda)\rangle_{0}=\frac{1}{1+\lambda e^{\sqrt{1-e^{2}} x_{0}}}|B\rangle .
$$

An alternative calculation of the boundary state of a rolling tachyon in a background electric field, involving a T-duality on $R$, a boost and another T-duality was given in [14] and gives the same result.

The origin of the matrix $M$ in (2.7) can be understood more clearly in the latter description: It merely reflects the Lorentz transformation on the pair $\left(X_{L}^{0}+X_{R}^{0}, X_{L}^{2}-X_{R}^{2}\right)$ since the T-dualization maps the electric field $e$ to the velocity $e$. Therefore, it is clear the oscillator part of the boundary state looks more or less the same as the case with $e=0$, once we use the co-moving coordinates in the T-dual description. In particular this implies that the oscillator part of the boundary state is as simple as before, if we concentrate on 
the part involving oscillators from 25 spatial directions in the co-moving frame of T-dual description. The latter can then be expressed in the following way:

$$
|B(\lambda)\rangle_{0}=\frac{i}{2} \sum_{w} \int d \mathcal{E} \frac{(2 \pi \lambda)^{i \mathcal{E}}}{\sinh \pi \mathcal{E}}\left|p_{L}^{0}=p_{R}^{0}=\sqrt{1-e^{2}} \mathcal{E}+e R w, p_{L}^{2}=-p_{R}^{2}=R w\right\rangle
$$

where we used

$$
\frac{1}{1+\lambda e^{x_{0}}}=\frac{i}{2} \int d \mathcal{E} \frac{(2 \pi \lambda)^{i \mathcal{E}}}{\sinh \pi \mathcal{E}} e^{i \mathcal{E} x_{0}}
$$

\section{Decay of D1 with electric flux}

The decay of the unstable brane into closed string modes has been discussed in [19]. Here, let us concentrate on the case of D1. The basic object is the one point function on the disk of a closed string state $\langle\mathcal{E}, w, N|$, with energy $\mathcal{E}$ winding number $w$ and left-right symmetric transverse oscillator excitations of level $N$,

$$
U\left(\mathcal{E}_{s}, w, k, N\right)=\left\langle\mathcal{E}_{s}, w, N \mid B\right\rangle=\sqrt{1-e^{2}}(2 \pi \lambda)^{i \mathcal{E}} \frac{1}{\sinh \pi \mathcal{E}}
$$

Where $\mathcal{E}$ appearing on the right hand side of (3.1) is related to the closed string energy $\mathcal{E}_{s}$ by

$$
\mathcal{E}=\frac{1}{\sqrt{1-e^{2}}}\left(\mathcal{E}_{s}-e R w\right)
$$

and $\mathcal{E}_{s}$ satisfies the usual mass shell condition

$$
-\mathcal{E}_{s}^{2}+(w R)^{2}+k_{\perp}^{2}+4(N-1)=0
$$

The total number of closed string states and the total energy produced in the decay of the brane is then given by

$$
\frac{\bar{N}}{V}=\sum_{\text {states }} \frac{1}{2 \mathcal{E}_{s}}\left|U\left(\mathcal{E}_{s}\right)\right|^{2}, \quad \frac{\bar{E}}{V}=\sum_{\text {states }}\left|U\left(\mathcal{E}_{s}\right)\right|^{2},
$$

where the sum goes over all on shell closed string states. Recall that, for a given level $N$, the number of closed string states with left and right oscillators matched grows exponentially in critical bosonic string theory for large level $N$ as

$$
c(N)=\frac{1}{\sqrt{2}} N^{-27 / 4} e^{4 \pi \sqrt{N}} .
$$


In the original calculation of the one point function in [19] a gauge was used [27] [28] that sets all timelike oscillators of the closed string states to zero. One important question is whether the presence of the electric field changes the large $N$ estimate (3.5) of the sum over states (3.4).

As far as the closed strings are concerned the boundary electric field is equivalent to a constant antisymmetric tensor background. For the non-zero mode excitations this leads to a chiral orthogonal rotation of right movers with respect to the left movers, resulting from the Lorentz transformation on $\left(X_{L}^{0}+X_{R}^{0}, X_{L}^{2}-X_{R}^{2}\right)$. The obvious choice of the gauge is then to adopt the same timelike gauge as in [19], except that now the timelike oscillators in the T-dual co-moving coordinate system are set to zero. This not only kills complications due to timelike oscillators in Sen' boundary state, as in [19, but also removes possible extra $e$-dependence that could have arisen from the matrix $M$. In the co-moving frame, $e$-dependence of $M$ is absorbed by the oscillator redefinition.

This way, the only net effect of $e$ in summing over the states will come from the zero mode part multiplied by simple exponential factor (3.5). With this, the total radiated energy for large $N$ behaves as

$$
\frac{\bar{E}}{V} \sim R\left(1-e^{2}\right) \sum_{w} \int d k_{\perp}^{24} \sum_{N} N^{-27 / 4} e^{4 \pi \sqrt{N}} e^{-\frac{2 \pi}{\sqrt{1-e^{2}}}\left(\sqrt{(w R)^{2}+k_{\perp}^{2}+4(N-1)}-e R w\right)},
$$

where we restored the normalization factor of $R$. In the sector with no winding $w=0$, (3.6) is exponentially suppressed because of the electric field. This implies that the amount of energy radiated away into unwound closed string is negligible [13] [29].

However if the winding modes are taken into account the picture changes drastically. Again with large $N$, the winding sectors such that

$$
w R \simeq 2 \frac{e \sqrt{N}}{\sqrt{1-e^{2}}}+\xi
$$

with small $\xi$ can be seen to contribute without exponential suppression. Expanding the exponent in (3.6)

$$
\begin{aligned}
& 4 \pi \sqrt{N}-\frac{2 \pi}{\sqrt{1-e^{2}}}\left(\sqrt{(w R)^{2}+k_{\perp}^{2}+4(N-1)}-e R w\right) \\
= & -\frac{\pi}{2 \sqrt{N}}\left(\left(1-e^{2}\right) \xi^{2}+k_{\perp}^{2}\right)+o\left(\frac{1}{N}\right)
\end{aligned} .
$$

4 This gauge choice is also justified indirectly by the cylinder amplitude of the next section. 
It is easy to see that the Hagedorn growth is cancelled by the exponential suppression coming from $e^{-2 \pi \mathcal{E}}$. In the spirit of large $N$ limit, we will replace the sums over $w$ and $N$ to integrals

$$
R \sum_{w} \sum_{N} \rightarrow \int d(w R) \int d N
$$

which in turn gives,

$$
\sum_{w} \int d k_{\perp}^{24} \sum_{N}=\int \frac{1}{2} \mathcal{E}_{s} d \mathcal{E}_{s} \int d k_{\perp}^{24} \int d(w R)
$$

Recall that along the saddle points

$$
\mathcal{E}_{s} \simeq \sqrt{(w R)^{2}+4(N-1)} \simeq \frac{2 \sqrt{N}}{\sqrt{1-e^{2}}}
$$

up to higher order corrections in $1 / N$. Then, the integrals become

$$
\left(1-e^{2}\right) \int \frac{1}{2} \mathcal{E}_{s} d \mathcal{E}_{s} \int d \xi \int d k_{\perp}^{24} N^{-27 / 4} e^{-\pi\left(\left(1-e^{2}\right) \xi^{2}+k_{\perp}^{2}\right) / 2 \sqrt{N}} .
$$

Evaluating this, we find all factors of $N$ and $\sqrt{1-e^{2}}$ drops out, and a simple integral emerges

$$
\frac{\bar{E}}{V} \sim \int d \mathcal{E}_{s}
$$

Note that, with the winding mode taken into account, the exponential suppression disappears and the total energy becomes linearly divergent. This is the same behavior as for the D0 brane in [19], and indicates that the energy of the decaying D1 branes (with electric field) goes mainly into highly wound strings.

To make contact with the low energy result described in section 1, let us note that the saddle point condition (3.7) can be written in terms of string energy as

$$
w R \simeq e \mathcal{E}_{s}
$$

which gives

$$
\frac{\text { string charge }}{\text { string energy }}=e \text {. }
$$

To summarize, we found that decay of D1 proceed primarily by many single string emissions and that individual strings emitted satisfy the above condition. This is a strong evidence that the unstable D1 with electric fields become a collection of strings with winding numbers in a manner precisely predicted by low energy approach. 
As an aside, let us discuss one obvious consequence in the decay process. Note that the width of the Gaussian distribution for $k_{\perp}$ is $N^{1 / 4}$, so the typical string has transverse velocity,

$$
\frac{\sqrt{\left\langle k_{\perp}^{2}\right\rangle}}{\mathcal{E}_{s}} \sim \frac{N^{1 / 4}}{\mathcal{E}_{s}} \sim\left(1-e^{2}\right)^{1 / 4} \frac{1}{\sqrt{\mathcal{E}_{s}}}
$$

which is suppressed by a factor of $\left(1-e^{2}\right)^{1 / 4}$ when string charge dominates the energy. Furthermore, since the winding energy is quantized in unit of $R$, the closed strings emitted tend to be rather heavy as $\mathcal{E}_{s}>|w||R| \geq|R| \gg 1$ when $R$ is large. Although the unstable D-brane is disintegrated into a number of such winding strings quickly, these closed strings take long time in "radiating" into the nearby spacetimes. The suppression comes primarily from having to radiate heavy strings with winding modes.

\section{Cylinder amplitudes}

By unitarity the total number of closed string states can be related to the imaginary part of a cylinder amplitude constructed from the boundary state. Although the open string interpretation and modular transformation properties of the full cylinder amplitude (including the real part) are still unclear it is useful to understand the absence of exponential suppression found in section 3 from this perspective. For the number of closed strings states emitted by a decaying brane one finds [19],

$$
\frac{\bar{N}}{V}=\sum_{\text {states }} \frac{1}{2 \mathcal{E}_{s}}\left|U\left(\mathcal{E}_{s}\right)\right|^{2}=\operatorname{Im}\left\langle B\left|\frac{b_{0}^{+} c_{0}^{-}}{2\left(L_{0}+\bar{L}_{0}-i \epsilon\right)}\right| B\right\rangle .
$$

Note that the dependence on the electric field $e$ in the cylinder amplitude comes purely from the zero mode contribution. This can be understood from the fact that the chiral rotation, discussed in section 3 , leaves $\bar{L}_{0}$ and hence the propagator on the right hand side of (4.1) invariant.

The one point function is given by (3.1) where $\mathcal{E}$ is given by (3.2).

$$
\begin{aligned}
\frac{\bar{N}}{V} & =\sum_{\text {states }} \sum_{w} \frac{1}{2 \mathcal{E}_{s}}\left|U\left(\mathcal{E}_{s}\right)\right|^{2} \\
& =R\left(1-e^{2}\right) \sum_{\text {states }} \sum_{w} \frac{1}{2 \mathcal{E}_{s}} \frac{1}{\pi \sinh ^{2}\left(\frac{\mathcal{E}_{s}+e R w}{\sqrt{1-e^{2}}}\right)}
\end{aligned}
$$


Where we separated the summation over the winding modes $w$ in the $X^{2}$ direction, from the sum over all physical states. (4.2) can be brought into a more convenient form by using the formulas

$$
\frac{1}{(\sinh \pi y)^{2}}=\sum_{n>0} 4 n e^{-2 \pi n y}
$$

and

$$
\begin{aligned}
\frac{1}{y} e^{-2 \pi y n} & =\frac{1}{\pi} \int d k_{0} \frac{1}{y^{2}+k_{0}^{2}} e^{2 \pi i k_{0} n} \\
& =\frac{1}{\pi} \int d k_{0} \int_{0}^{\infty} d t e^{-t\left(k_{0}^{2}+y^{2}\right)} e^{2 \pi i k_{0} n} .
\end{aligned}
$$

Putting everything together (4.2) becomes

$$
\frac{\bar{N}}{V}=\frac{2 R}{\pi^{2}} \sqrt{1-e^{2}} \sum_{\text {states }} \sum_{w} \sum_{n>0} n e^{2 \pi n \frac{e R w}{\sqrt{1-e^{2}}}} \int d k_{0} \int d t e^{-t\left(k_{0}^{2}+\frac{\mathcal{E}_{s}^{2}}{1-e^{2}}\right)} e^{2 \pi i k_{0} n} .
$$

Where $\mathcal{E}_{s}$ is given by (3.3). The Gaussian integrals over $k_{0}$ and the twenty four transverse momenta $k_{\perp}$ give

$$
\frac{\bar{N}}{V}=\frac{2 R}{\pi}\left(1-e^{2}\right) \sum_{\text {states }} \sum_{w} \sum_{n>0} n e^{2 \pi n \frac{e R w}{\sqrt{1-e^{2}}}} \int d \tilde{t} \tilde{t}^{-25 / 2} e^{-\frac{\pi n^{2}}{\tilde{t}\left(1-e^{2}\right)}} e^{-\tilde{t}\left((w R)^{2}+4(N-1)\right)}
$$

where we have rescaled $t=\pi\left(1-e^{2}\right) \tilde{t}$. The sum over winding modes can be rewritten using the Poisson resummation formula

$$
\sum_{k} e^{-\pi a k^{2}+2 \pi i b k}=\frac{1}{a^{\frac{1}{2}}} \sum_{m} e^{-\frac{\pi(m-b)^{2}}{a}}
$$

The summation over transverse string oscillators produces

$$
\frac{\bar{N}}{V}=\frac{2}{\pi}\left(1-e^{2}\right) \sum_{k, n>0} n \int \frac{d \tilde{t}}{\tilde{t}^{13}} \frac{e^{4 \pi \tilde{t}}}{\prod_{m}\left(1-e^{-2 \pi \tilde{t} m}\right)^{24}} e^{-\frac{\pi}{\tilde{t}} \frac{n^{2}}{1-e^{2}}} e^{-\frac{\pi^{2}}{t} \frac{1}{R^{2}}\left(k-\frac{i e R n}{\sqrt{1-e^{2}}}\right)^{2}} .
$$

A modular transformation $s=\frac{1}{2 t}$ expresses (4.8) as a open string partition function.

$$
\frac{\bar{N}}{V}=\frac{2^{13}}{\pi}\left(1-e^{2}\right) \sum_{n>0, k} \int \frac{d s}{s} \frac{1}{\prod_{m}\left(1-e^{-\pi s m}\right)^{24}} e^{-\pi s\left(-1+n^{2}+\frac{k^{2}}{R^{2}}\right)} .
$$

Note that the absence of an exponential suppression in the ultraviolet $(s \rightarrow \infty)$ region of the integral since the exponentially growing term in the exponent of (4.9) is cancelled by the $n=1$ term precisely. Note that the cylinder amplitude also gives an indication that analysis given in section 3 is correct. A electric field dependent change in (3.4) and (3.5), that changes the saddle point calculation given in section 3, should also invalidate the cancellation in (4.9), and this does not happen. 


\section{Summary}

We studied closed string one point amplitude on the boundary state of unstable Dbranes. Wrapping a D1 on a circle, and turning on electric field along the circle, we find that closed strings produced by the decay are primarily given by certain winding strings with a definite ratio between the winding energy and the oscillator energy and that all energy of the initial system is accounted for by this decay channel.

This indicates that the final state of unstable D-brane in the tree-level approximation is really made of closed strings with winding number, in accordance with previous expectation based on low energy approaches. In terms of languages of the latter, the combined system of string fluid and tachyon matter is really a macroscopic collection of stretched fundamental strings with high frequency oscillations moving along the length. While the one-point amplitude is most telling for case of unstable D1, we expect that the same closed string interpretation will work for higher dimensional D-branes. In the large radius limit, this also implies that produced strings are extremely heavy and do not easily disperse into the neighboring spacetime.

\section{Acknowledgment}

Both authors are grateful to the theory group at CERN for hospitality during the 'Strings at CERN' workshop. The work of MG is supported in part by NSF grant PHY0245096. Any opinions, findings and conclusions expressed in this material are those of the authors and do not necessarily reflect the views of the National Science Foundation. 


\section{References}

[1] A. Sen, "Tachyon condensation on the brane antibrane system," JHEP 9808, 012 (1998) arXiv:hep-th/9805170.

[2] P. Yi, "Membranes from five-branes and fundamental strings from Dp branes," Nucl. Phys. B 550, 214 (1999) arXiv:hep-th/9901159.

[3] O. Bergman, K. Hori and P. Yi, "Confinement on the brane," Nucl. Phys. B 580, 289 (2000) arXiv:hep-th/0002223.

[4] G. W. Gibbons, K. Hori and P. Yi, "String fluid from unstable D-branes," Nucl. Phys. B 596, 136 (2001) arXiv:hep-th/0009061.

[5] A. Sen, "Fundamental strings in open string theory at the tachyonic vacuum," J. Math. Phys. 42, 2844 (2001) arXiv:hep-th/0010240.

[6] J. McGreevy and H. Verlinde, "Strings from tachyons: The $\mathrm{c}=1$ matrix reloaded," JHEP 0312, 054 (2003) [arXiv:hep-th/0304224.

[7] T. Takayanagi and N. Toumbas, "A matrix model dual of type 0B string theory in two dimensions," JHEP 0307, 064 (2003) arXiv:hep-th/0307083.

[8] M. R. Douglas, I. R. Klebanov, D. Kutasov, J. Maldacena, E. Martinec and N. Seiberg, "A new hat for the $\mathrm{c}=1$ matrix model," arXiv:hep-th/0307195.

[9] I. R. Klebanov, J. Maldacena and N. Seiberg, "D-brane decay in two-dimensional string theory," JHEP 0307, 045 (2003) arXiv:hep-th/0305159.

[10] A. Sen, "Rolling tachyon," JHEP 0204, 048 (2002) arXiv:hep-th/0203211.

[11] A. Sen, "Tachyon matter," JHEP 0207, 065 (2002) arXiv:hep-th/0203265.

[12] M. Gutperle and A. Strominger, "Spacelike branes," JHEP 0204, 018 (2002) arXiv:hep-th/0202210.

[13] P. Mukhopadhyay and A. Sen, "Decay of unstable D-branes with electric field," JHEP 0211, 047 (2002) arXiv:hep-th/0208142.

[14] S. J. Rey and S. Sugimoto, "Rolling tachyon with electric and magnetic fields: Tduality approach," Phys. Rev. D 67, 086008 (2003) arXiv:hep-th/0301049.

[15] B. Chen, M. Li and F. L. Lin, "Gravitational radiation of rolling tachyon," JHEP 0211, 050 (2002) arXiv:hep-th/0209222.

[16] M. Gutperle and A. Strominger, "Timelike boundary Liouville theory," Phys. Rev. D 67, 126002 (2003) arXiv:hep-th/0301038.

[17] T. Okuda and S. Sugimoto, "Coupling of rolling tachyon to closed strings," Nucl. Phys. B 647, 101 (2002) arXiv:hep-th/0208196.

[18] N. R. Constable and F. Larsen, "The rolling tachyon as a matrix model," JHEP 0306, 017 (2003) arXiv:hep-th/0305177.

[19] N. Lambert, H. Liu and J. Maldacena, "Closed strings from decaying D-branes," arXiv:hep-th/0303139. 
[20] A. Sen, "Dirac-Born-Infeld action on the tachyon kink and vortex," Phys. Rev. D 68, 066008 (2003) arXiv:hep-th/0303057.

[21] A. Sen, "Field theory of tachyon matter," Mod. Phys. Lett. A 17, 1797 (2002) arXiv:hep-th/0204143.

[22] G. Gibbons, K. Hashimoto and P. Yi, "Tachyon condensates, Carrollian contraction of Lorentz group, and fundamental strings," JHEP 0209, 061 (2002) arXiv:hepth/0209034.

[23] O. K. Kwon and P. Yi, "String fluid, tachyon matter, and domain walls," JHEP 0309, 003 (2003) arXiv:hep-th/0305229.

[24] A. Strominger, "Open string creation by S-branes," arXiv:hep-th/0209090.

[25] A. Sen, "Open-closed duality at tree level," Phys. Rev. Lett. 91, 181601 (2003) arXiv:hep-th/0306137.

[26] H. U. Yee and P. Yi, "Open / closed duality, unstable D-branes, and coarse-grained closed strings," Nucl. Phys. B 686, 31 (2004) arXiv:hep-th/0402027.

[27] S. Hwang, "No Ghost Theorem For SU(1,1) String Theories," Nucl. Phys. B 354, 100 (1991).

[28] J. M. Evans, M. R. Gaberdiel and M. J. Perry, "The no-ghost theorem for AdS(3) and the stringy exclusion principle," Nucl. Phys. B 535, 152 (1998) arXiv:hepth/9806024.

[29] K. Nagami, "Closed string emission from unstable D-brane with background electric field," JHEP 0401, 005 (2004) arXiv:hep-th/0309017. 